\title{
A Comparison of Distance Fly Casting Performance Between Three 5- Weight Long-belly Floating Lines
}

\author{
Ulrik Röijezon ${ }^{1, *}$ and Stefan Siikavaara ${ }^{2}$ \\ ${ }^{I}$ Department of Health Sciences, Luleå University of Technology, 97187 Luleå, Sweden \\ ${ }^{2}$ Dala Sports Academy, University of Dalarna, 79188 Falun, Sweden
}

\begin{abstract}
Long-belly fly lines have gained extensive popularity around the world, both for fishing and for competitive distance casting. In Sweden, three long-belly fly lines are used as official lines in Fly Casting competitions: The Mastery Expert Distance (Scientific Anglers, USA), the Rio Gold Tournament (Rio, USA) and the Barrio GT140 (Barrio Fly Lines, Scotland). The objective of this study was to compare distance casting performance between the three long-belly fly lines using a standardized test protocol. Sixteen elite casters, eight competitors in Fly Casting and eight competitors in Casting, each performed eight casts with all three lines. All lines were cast in a randomized manner with the same type of rod (MSX Sapphire 790-4) fitted with the same type of reel and identical leaders. The test was performed as an indoor competition using the overhead cast technique. The statistical analyses revealed no significant differences between the lines. This result was consistent both for the whole group $(\mathrm{p}>0.05)$ and when the results from the subgroups of competitors in Fly Casting and Casting were analyzed separately $(p>0.05)$. The conclusion of the study is that at group level there are no significant differences in distance casting performance between the three lines when casting is performed indoors. At an individual level, however, some casters may have preferences for a specific line. These preferences may be related to factors such as the familiarity with the line, or how well it suits an individual casting technique.
\end{abstract}

Keywords: Competition, Fly Casting, Fly fishing, Fly line, Long-belly, Test, Trout-Distance.

\section{INTRODUCTION}

Recreational sport fishing, including fly fishing, is a highly popular outdoor leisure activity in many areas around the world [1]. From the leisure activity of fishing and fly fishing, the sports Tournament Casting and Competition Fly Casting have evolved. These two sports will from here on be referred to as Casting and Fly Casting, respectively. Casting involves distance and accuracy casting with spinning and fly rods and lines, and has been an established sport since at least 1957, when the first world championship tournament was conducted in Kiel, Germany. The fly rods and lines used in Casting are specifically designed for casting competition and have little, or no, use in fly fishing situations. Fly Casting, on the other hand, involves regular fly fishing equipment and has a relatively young history as an established sport. The first world championship in Fly Casting was held in Fagernes, Norway, 2010. Fly Casting includes six different events, where the goal is to cast either as accurate or as far as possible. Three events involve casting with single-handed rods while the other three events involve casting with two-handed rods.

Fly Casting is, in contrast to Casting, aimed to remain its close relation to fishing, and most competitors are fly fishers

*Address correspondence to this author at the Department of Health Sciences, Luleå University of Technology, 97187 Luleå, Sweden;

Tel: +46 (0) 920-49 29 87; Fax: +46 (0) 920-49 13 99;

E-mail: ulrik.roijezon@ltu.se who have taken up the sport due to their interest in fly fishing. The regulations regarding the equipment used in the various events are therefore set to be closely related to actual fishing situations. The equipment regulations concern for example the length of the fly rods, and the type or the weight of fly lines used.

The development of fly lines for single-handed rods, with the aim to reach long distance in fly fishing, has roughly taken two different directions. One direction involves relatively short and heavy tapers, so called shooting-heads, which commonly are combined with a very thin shooting line characterised by a minimal friction. This design allows for long casts without carrying long line during false casting before the delivery cast. Shooting-heads are commonly used in large waters, such as the sea and in big rivers or lakes, when fishing with large flies. Within Fly Casting, shootinghead lines are used in the event Sea-Trout Distance. The other direction of fly line development involves long tapers, i.e. long-belly lines. These line designs allow for a larger variation regarding the use of type and size of the fly, as well as fishing short and long distances with discreet presentations of the fly. This type of line is used in the event Trout Distance in Fly Casting. Opposite to the shooting-head design, the long tapers require long line carrying during false casting before the delivery cast in order to get proficient weight, and thereby kinetic energy, into the line when performing long distance casts. 
Over the last 10-15 years the Mastery Expert Distance (MED) fly line made by Scientific Anglers TM (SA), USA has been one of the most used long-belly fly lines worldwide. Apart from being a popular fishing line, the SA MED was used as the official line in the World Championship in Fly Casting 2010, in Fagernes, Norway, in the Trout Distance and Trout Accuracy events. Recently two new fly lines with long-belly weight forward tapers have been introduced on the market: the Rio Gold Tournament (Rio, USA) and the Barrio GT140 (Barrio Fly Lines, Scotland). Both these lines have gained large approval among fly fishers and fly casters and have become established fly lines worldwide. These two lines were 2011 introduced as official lines, together with the SA MED, in the event Trout Distance at official competitions in Sweden, including the Open Nordic Championship in Göteborg 2011 and the Swedish Championship in Pitea 2012. Although the three lines are all categorized as long-belly floating fly lines, they do have some slight differences in their designs which influence their casting characteristics. For example, the SA MED has a belly of about 21 meters (m), the Rio Gold Tournament has a $23 \mathrm{~m}$ long belly and a very thin running line, while the Barrio GT140 has the longest belly reaching $27 \mathrm{~m}$, but compared to the other lines, a thicker running line. Other differences between these lines include the taper design of the different parts of the line belly as well as the materials used in making the lines.

The techniques used to carry long line in false casting, which involve, e.g., long casting strokes and high line speed, make the design of the fly line highly important to maximise casting distance. For example should the belly of the line be adequately long to allow for the carry of as long line as possible with as high speed as possible before delivery, and the running line section should contribute with as small amount of resistance (friction) as possible. Also, the air resistance should be small to minimise loss of kinetic energy during the backward, forward and delivery phases of the cast. The question regarding which long-belly fly line is the best line for distance casting has been debated among fly fishers and fly casters, both in personal communications and on various forums on the internet. Is there an "optimal formula" for the long-belly fly line design or is the performance more related to personal preferences and casting techniques? Since new lines have been introduced to the market, each with its specific design, it would be valuable to compare these lines against each other using a standardized test protocol.

Research on physics of fly casting has been published in previous papers [2-9]. Some of these studies target the dynamics of the fly line [5-7], dynamics of the fly rod [9] and the motion of the fly rod during casting and even robotics performing fly casting [8]. One of the future aims of these studies is to model and assess the performance of equipment, e.g., fly lines, in standardized manner. So far, these studies have contributed with important knowledge about the physics and dynamics of fly casting, especially in short and medium long casts with level or double taper. However, to date, no models or robots have been described to evaluate the performances of various taper designs in maximum distance casting. Therefore, the best way to evaluate this is by collecting empirical data from fly casters casting for maximum distance.

In the present study we assessed the performance of the three long-belly floating lines which are accepted as official fly lines in the event Trout Distance in Fly Casting competitions in Sweden. The lines are also used as fishing lines by fly fishers around the world. The test was executed in an indoor setting using a standardized test protocol. Each line was cast with the same type of fly rod, reel and leader by a group of elite casters in Fly Casting and a group of casters with less experience of casting long-belly fly lines, but who are elite casters in Casting. The main aim of the study was to compare maximum distance casting performance between the three fly lines. A secondary aim was to investigate if there are differences in the fly lines performances among fly casters who have a large experience using long-belly fly lines and among casters who have less, or no, experience with long-belly fly lines, but competes in Casting.

\section{METHOD}

\section{Participants}

Sixteen casters performed the fly line test, ages ranging from 19 to 55 years. Eight of the participants had experience in Fly Casting competitions and were thereby used to cast long-belly lines. Eight had less, or no, experience in longbelly fly casting, but were competitors in Casting. Two women participated, one with experience in Fly Casting and one with experience in Casting. Four of the casters were Certified Casting Instructors (CCI), of which two were Master Certified Casting Instructors (MCCI), within the Federation of Fly Fishers (FFF). Oral informed consent was obtained from all casters before the test.

\section{Equipment and Procedure}

Three long-belly floating lines, all weight 5 according to the standards of American Fly Fishing Trade Association (AFFTA), were used in the test. The test was arranged as a distance casting competition. The fly lines included were the Barrio GT140 (Barrio Fly Lines, Scotland), the Rio Tournament (RIO, USA) and the Mastery Expert Distance (MED) (Scientific Anglers TM, USA). All lines had a similar orange color. Three fly rods were used to allow for simultaneous casting with the three lines. The fly rods were all the same model (Sapphire 790-4, MSX Fly Fishing, Sweden) and identical fly reels (Danielsson/Loop Graphite, Loop, Sweden) were used for each of the three lines to avoid influences in casting performance related to the equipment used. A standardized $2.7 \mathrm{~m}$ leader was connected to each of the lines and a small piece of yarn was used as a fly. The leaders where tied together using $1.35 \mathrm{~m}$ of $0.40 \mathrm{~mm}, 0.75 \mathrm{~m}$ of $35 \mathrm{~mm}$ and $0.60 \mathrm{~m}$ of $0.30 \mathrm{~mm}$ nylon tippet.

One cast was allowed with each line before the test so the caster could become familiar with the lines. For the test, each participant was allowed eight casts with each line. The task was to cast as far as possible.

The lengths of the casts were measured along measure tapes placed on the ground and all eight casts were recorded for each caster. The participants were unaware of which of the three lines that were tackled on to each of the rods: i.e. the participants were blind to the line used on the different 


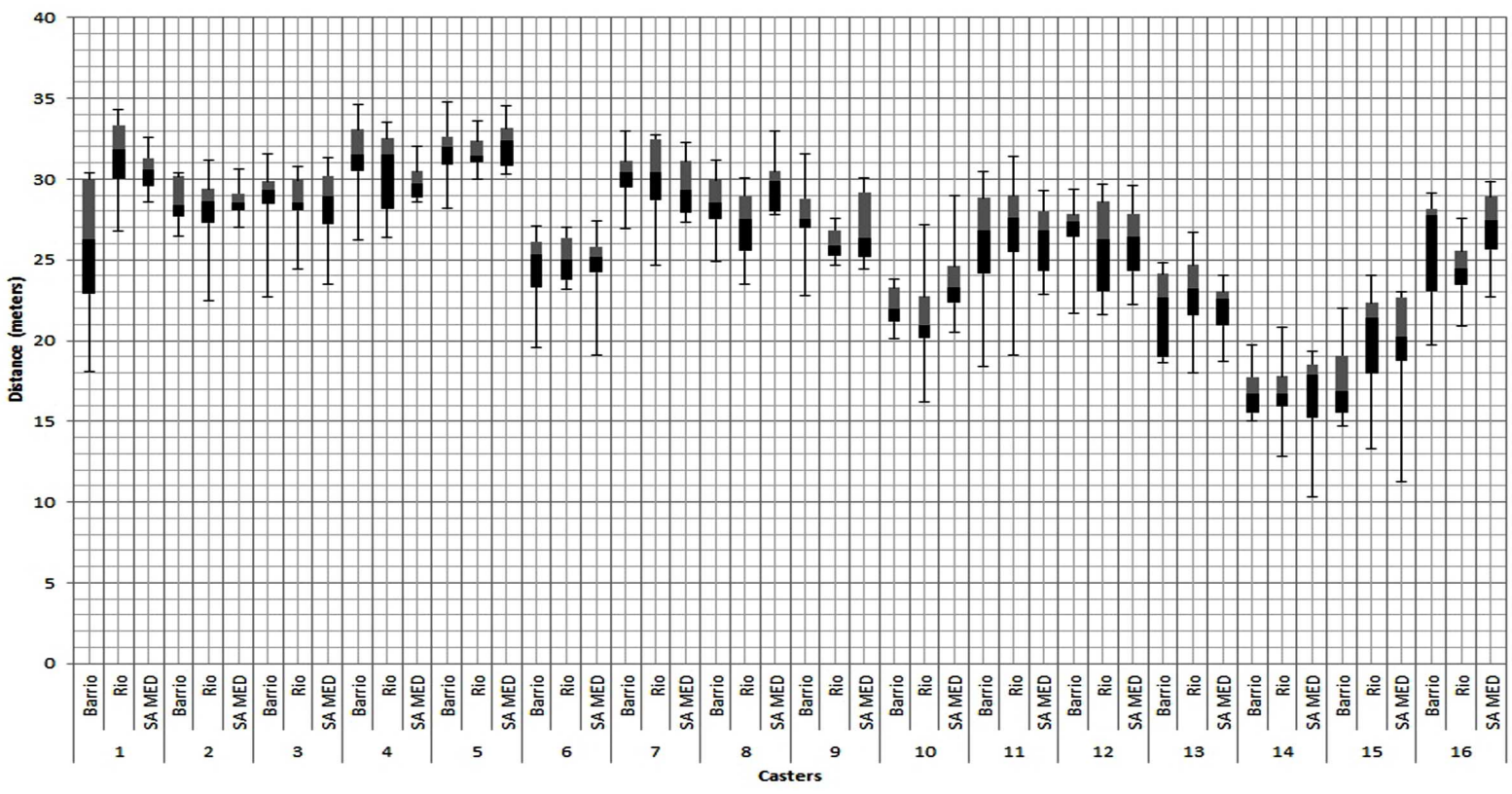

Fig. (1). Box-plot illustrating the distances casted with each AFFTA 5-weight long-belly fly line, Barrio GT140 (Barrio), Scientific Anglers Mastery Expert Distance (SA MED) and Rio Gold Tournament (Rio), for each caster. The upper and lower whiskers represent the longest and shortest casts, respectively. The box represents the median, and the $1^{\text {st }}$ and $3^{\text {rd }}$ quartiles. Caster 1-8 are competitors in Fly Casting, while caster $9-16$ are competitors in Casting.

rods during the competition. The casting procedure was performed by the casters in a random order.

The competition took place on an indoor football field between 8.30 and 11.30 in the morning in November 2010, at the Bosön Sports Camp, Stockholm, Sweden. Temperature was $16^{\circ} \mathrm{C}$ and air moisture was $40-50 \%$.

\section{Data Analyses}

Descriptive statistics, including maximum and mean values and standard deviations (SD), were calculated for the eight casts for each participant and fly line. The mean values and SDs of the longest casts (i.e., the maximum values) as well as the mean value and SD for each caster and line were calculated for the whole group of casters, and for each subgroup of casters. That is, the subgroups of competitors in Fly Casting and competitors in Casting. SPSS 19.0 for windows was used for statistical analyses. Analysis of Variance (ANOVA) was used for analyses of differences in casting distance performance between the fly lines. A fullfactorial ANOVA with line-type and caster, and their interaction, as factors was performed for the complete group of casters. Univariate ANOVAs were performed to investigate possible differences for the longest casts, mean values and SD between the lines for the whole group, as well as for the separate subgroups of competitors in Fly Casting and Casting. A p-value $<0.05$ was considered significant.

\section{RESULTS}

The two longest casts were performed with the Barrio GT140 (34.8 $\mathrm{m}$ and $34.6 \mathrm{~m}$ ), the third longest cast was performed with the SA MED (34.5 m) and the fourth longest cast was performed with the Rio Tournament (34.3 m), see Fig. (1).

The longest and shortest casts, as well as median and $1^{\text {st }}$ and $3^{\text {rd }}$ quartiles, for each line and caster are presented in Fig. (1).
The full-factorial ANOVA with line and caster as factors revealed that there are no differences in casting performance between the lines for this group of casters $F=0.9(p=0.41)$. There is a significant difference between casters $F=61.9$ $(\mathrm{p}<0.001)$, but no significant interaction between line and caster $\mathrm{F}=1.4(\mathrm{p}=0.092)$.

Table 1 presents the mean values (and SD) of the longest casts, mean distances and SDs for each line and caster for the whole group of casters $(n=16)$, and for the two sub groups of casters separately, i.e., Fly Casting competitors $(n=8)$ and Casting competitors $(\mathrm{n}=8)$. No significant difference appeared between the lines for the longest casts, mean distances or SD for the whole group of casters $(p>0.05)$. Nor were any significant differences detected when the results of competitors in Fly Casting and competitors in Casting were analyzed separately $(\mathrm{p}>0.05)$.

\section{DISCUSSION}

The aim of the study was to compare maximum distance casting performance between three long-belly floating lines AFFTA weight 5: Barrio GT140, Rio Gold Tournament and SA MED. The two longest casts were performed with the Barrio line, the third longest with the SA line and the fourth longest with the Rio line. There were, however, no significant differences found between the lines, neither in the full-factorial model, or for the longest casts, mean distances and standard deviations. These non-significant results were consistent both for the whole group and when the two separate subgroups of experienced and less experienced fly casters were analyzed separately. The fact that no significant differences were found for either of the outcome variables indicates that there is no general advantage for a specific line, at least not when casting indoors with the specific type of rod used in the present study. 
Table 1. Distance casting Performance of the AFFTA 5-Weight Long-Belly Floating Lines Barrio GT 140, Rio Gold Tournament and Scientific Anglers Mastery Expert Distance for the Entire Group of Casters, and the Subgroups of Competitors in Fly Casting and Casting

\begin{tabular}{|c|c|c|c|c|c|c|}
\hline & & Barrio & Rio & SA MED & F -value \\
\hline \hline \multirow{3}{*}{ All casters (n=16) } & Longest & $29.0 \pm 4.4$ & $29.3 \pm 3.7$ & $29.2 \pm 4.1$ & 0.020 & 0.980 \\
\cline { 2 - 7 } & Mean & $25.8 \pm 4.4$ & $26.0 \pm 4.2$ & $26.3 \pm 4.2$ & 0.055 & 0.946 \\
\cline { 2 - 7 } & SD & $2.6 \pm 0.9$ & $2.5 \pm 0.8$ & $2.3 \pm 0.8$ & 0.528 & 0.593 \\
\hline \multirow{3}{*}{$\begin{array}{c}\text { Competitors in Fly } \\
\text { Casting (n=8) }\end{array}$} & Longest & $31.6 \pm 2.5$ & $31.7 \pm 2.4$ & $31.7 \pm 2.1$ & 0.002 & 0.111 \\
\cline { 2 - 7 } & Mean & $28.7 \pm 2.5$ & $29.1 \pm 2.3$ & $29.2 \pm 2.2$ & 2.012 & 0.998 \\
\hline \multirow{3}{*}{$\begin{array}{c}\text { Competitors in } \\
\text { Casting (n=8) }\end{array}$} & SD & $2.5 \pm 0.9$ & $2.2 \pm 0.6$ & $1.8 \pm 0.6$ & 0.159 \\
\cline { 2 - 7 } & Longest & $26.4 \pm 4-4$ & $26.9 \pm 3.3$ & $26.8 \pm 4.1$ & 0.037 & 0.963 \\
\cline { 2 - 7 } & SD & $23.0 \pm 4.2$ & $23.0 \pm 3.5$ & $23.5 \pm 3.9$ & 0.042 & 0.959 \\
\hline
\end{tabular}

Mean \pm SD distance in meters for the longest casts (Longest) and for the mean distances (Mean) and standard deviations (SD) of the eight casts by each caster. Barrio GT 140 (Barrio), Rio Gold Tournament (Rio) and Scientific Anglers Mastery Expert Distance (SA MED).

Distance casting with long-belly fly lines involves carrying long line during false casting in order to get a large mass moving and thereby generate a high kinetic energy in the line. The ability to carry a long line is therefore related to the ability to cast long distances. Since the length of the bellies of the lines varied from $21 \mathrm{~m}$ (SA MED) to $27 \mathrm{~m}$ (Barrio GT140) it seemed possible that the shortest belly would suit less experienced fly casters best, while the experienced fly casters could have an advantage casting lines with the longest bellies. However, the results revealed no difference between the lines for any of the outcome variables when the experience and less experienced groups were analyzed separately. A possible explanation is that the experienced fly casters have most experience casting the SA MED. This may be partly supported by the results showing a tendency (although not significant) towards a smaller variability (standard deviation) of the casts with the SA MED, compared to the cast with the newer lines, in the group of experienced casters, see Table 1. It is possible that increased practice with the new lines will lead to adaptations and improvements in casting techniques that will see future gains for these newer lines. On an individual level a caster may prefer a specific line over the others. Although no significant interaction was found for caster and line, Fig. (1) does show that some of the casters vary in their casting performance between the different lines.

In this study the equipment was highly standardized, using the same type of rod, reel and leader. Moreover, the casting was performed indoor and all three lines were cast simultaneously by the different casters in a randomized manner. Together, this procedure reduces the risk of influence on the results from factors other than the performance of the line and the performance of the casters.

This study was limited to comparing the three lines in distance casting under controlled indoor conditions. Therefore these results cannot give any information about performances in outdoor conditions, when using other types of casts or casting with different types of rods. In fishing and outdoor casting competitions the weather conditions, including wind, air moisture and temperature, have a considerable influence on the performance of fly lines. It is possible that the different lines have different advantages for example when casting with the wind from the back compared to casting in head or side wind. Moreover, it is possible that the different lines have different advantages when performing alternative casts often used in fishing, such as roll, switch and spey casts as well as various presentations casts, e.g., curve and mend casts. Lastly, it is also possible that each of the different lines performs best with a specific type of rod, e.g., the Barrio GT140 has the longest belly and therefore also the highest weight when carrying long line ( $>$ $20 \mathrm{~m}$ ) during false casting. It may therefore be possible that casting the Barrio GT140 works better with a relatively stiffer rod compared to casting the SA MED or Rio Gold Tournament.

\section{CONCLUSION}

The longest casts were performed with the Barrio GT140. However, the results imply that there are no differences in distance casting performance between the lines at a group level. At least no significant differences when casting is performed indoors with the overhead casting technique with the specific rod used in this study. On an individual level, however, a caster may prefer a specific line. This may be related to the familiarity with the line, or how well it suits the casting technique of that individual.

\section{CONFLICT OF INTEREST}

There are no economical or other interests between the authors and the manufacturers of the fly lines assessed in the present study. The fly rods used in the study where sponsored by MSX Fly Fishing, a Swedish company to whom the authors are connected as Pro Staffs.

\section{ACKNOWLEDGEMENTS}

The authors would like to thank the Swedish Casting Association for their help with completing the study, Magnus Angus for valuable help reviewing the manuscript and Bernt Johansson at Klikki AB for graphical work. Also thanks to MSX Fly Fishing for sponsoring of fly rods. 


\section{REFERENCES}

[1] Arlinghaus R, Cooke SJ. Recreational fisheries: socioeconomic importance, conservation issues and management challenges. In: Dickson B, Hutton J, Adams WM, Eds. Recreational hunting conservation and rural livelihoods: science and practice. Oxford: Blackwell Publishing 2009; pp. 39-58.

[2] Spolek GA. The mechanics of fly casting - the fly line. Am J Phys 1986; 54(9): 832-6.

[3] Lingard S. Note on the aerodynamics of a flyline. Am J Phys 1988; 56(8): 756-7.

[4] Robson JM. The physics of fly casting. Am J Phys 1990; 58(3): 234-10.
[5] Gatti-Bono C, Perkins NC. Physical and numerical modelling of the dynamic behavior of a fly line. J Sound Vib 2002; 255(3): 55577.

[6] Gatti-Bono C, Perkins NC. Effect of loop shape on the draginduced lift of fly line. J Appl Mech Trans ASME 2004; 71(5): 745-7.

[7] Gatti-Bono C, Perkins NC. Numerical model for the dynamics of a coupled fly line/fly rod system and experimental validation. J Sound Vib 2004; 272(3-5): 773-91.

[8] Anderson D., Perkins NC. Richards B. Quantitative understanding of the fly casting stroke through measurements and robotic casting. Sports Eng 2006; 9: 97-106.

[9] Wang G, Wereley N. Analysis of fly fishing rod casting dynamics. Shock Vib 2011; 18(6): 839-55.

(C) Röijezon and Siikavaara; Licensee Bentham Open.

This is an open access article licensed under the terms of the Creative Commons Attribution Non-Commercial License (http://creativecommons.org/licenses/ by-nc/3.0/) which permits unrestricted, non-commercial use, distribution and reproduction in any medium, provided the work is properly cited. 